3. Already damaged tissues are again bruised, and their resistance to infection is further lowered.

Where a scleral route is adopted, the advantages are:

1. The operation is easier to perform, for the magnet is nearer, and so more efficient, and there is no resistance to the passage of the foreign body. The vitreous is disturbed as little as possible.

2. The inflammatory exudate may even come out with the foreign body.

3. The ciliary segment is not bruised.

4. A sutured conjunctival wound has much greater freedom from late infection than a corneal wound.

The disadvantages of the scleral wound is:-impaction of the choroid, retina, and vitreous in the wound. This very rarely occurs; I have found it in one or two cases where I have had to excise the eye eventually.

I claim the following advantages:-

(1) A larger percentage of eyes is saved. (2) A better visual acuity in the saved eye. (3) A shorter period of convalescence after operation.

\title{
THE TECHNIQUE OF THE HAAB AND SMALL MAGNETS IN THE EXTRACTION OF INTRA-OCULAR FOREIGN BODIES.
}

\author{
BY \\ Maurice, H. Whiting, and Charles Goulden, \\ CAPTAIN R.A.M.C. (TEMP.) LIEUTENANT R.A.M.C. (TEMP.)
}

DURING the past twelve months we have dealt at the Hospital to which we are attached with a great number of perforating wounds of the eye.

Many of these eyes contained magnetic foreign bodies.

Some idea of the number of such cases will be gathered from the fact that in July, 1916, alone, there were 30 cases in which magnetic foreign bodies were removed from within the globe; in one day, 5 such cases were operated upon, and more recently, 13 in one week.

With such a large amount of material, it has been our endeavour to work out some systematic plan of treatment applicable to the various conditions found ; and in the course of our operations we

- have elaborated many small points of technique, to overcome the varied difficulties encountered.

We are not aware that any detailed description of the use of the 
Haab and small magnets in combination has been published, and it is the object of this paper to attempt to supply that deficiency.

The methods used are, in the main, an elaboration of those we have always used at Moorfields, and later (by one of us) at Oldham.

Cases of magnetic foreign bodies within the globe are comparatively rare in civil life, and any one operator, with few exceptions, does not deal with a great number in a single year. For instance, at Moorfields there were, between 1901 and 1905 inclusive, but 118 cases, although we are aware that this number has been exceeded in recent years, and that such cases must be more frequent in manufacturing areas where there are many iron and steel industries.

General principles of method adopted.-The wound of entry made by the foreign body, be it corneal or scleral, is disregarded from the point of view of extraction, unless there be a large unhealed wound in the cornea or sclera. Such a wound indicates the presence of a large foreign body in the vitreous, which it would be hazardous to remove by any other path. In any case these eyes are so severely damaged that there is but little chance of saving them.

The method we adopt is to draw the foreign body forward through the suspensory ligament of the lens into the posterior chamber, and then through the pupil into the anterior chamber. This operation is performed with the Haab magnet.

The foreign body is then removed from the anterior chamber, through a suitable corneal incision, by means of the small magnet.

The alternative to this general method is to remove the foreign body, after localization by X-rays, through the original scleral wound, or an enlargement of it, or through a suitably placed puncture in the sclera. In France, where a considerable number of the foreign bodies are non-magnetic, this would entail in many cases an unnecessary opening in the eye.

In a paper published by one of us in the Royal London Ophthalmic Hospital Reports, Vol. XVII, Part ii, it was shown that the afterresults in civil cases treated by this method, were not so good as those in which the foreign body was removed via the pupil and anterior chamber.

The assistance given by an X-ray localization is by no means essential to successful treatment; if the localization is likely to involve more than two or three hours' delay, it should be omitted.

Description of apparatus employed.-The giant magnet we use is the most recent type designed by Professor Haab, and supplied from $Z$ urich. It is hooded, actuated by a pedal, and has no rheostat. It is fitted with a chin rest, which we have removed, as we consider it hampers movement of the patient's head. Of the various terminals supplied, we use only the medium sharp-pointed short one, which gives a maximum of power with a minimum obstruction to view. Variation of power, when required, is obtained by advancing 
the eye to or withdrawing the latter from the point of the magnet. The terminal is sterilized by boiling before use. The small magnet is one supplied by Messrs. Weiss, weighing about half a pound, and worked from a 3 or 4 cell accumulator. The terminal is about 1 inch long, of soft iron, originally cylindrical, but hammered, so as to become oval in section, and tapered like a screwdriver, and afterwards polished. This is sterilized by boiling before use, and the body of the magnet is wrapped in sterile gauze.

The corneal section is made with a straight keratome, $10 \mathrm{~mm}$. from base to apex and $8 \mathrm{~mm}$. in its broadest part. This enables one to make the section sufficiently large by an onward thrust without lateral movement. The importance of this will be seen later on.

Lang's solid blade speculum, fixation forceps (2 teeth into 3), Lang's double-curved iris forceps, de Wecker's scissors, and iris repositor complete the outfit.

When it is found necessary to wash out the anterior chamber, we use an undine with 3 feet of rubber tubing and a flattened silver nozzle.

For illuminating the eye in the various steps of the operation, we have found Curry and Paxton's operating lamp with Nernst burner most useful.

Preparation of patient for operation.-The patient, if possible, should be prepared as for a general anæsthetic, by having the bowels and bladder emptied and no food given for 3 or 4 hours beforehand. The face and lids should be cleansed with a simple lotion, such as saline or boric solution. It is most important to have the pupil well dilated with atropine; a sterile pad is then applied. In some cases it may not be possible to fulfil all these conditions, when operative treatment is urgently required, but the pupil must be dilated.

Immediately before operation, two applications of 2 per cent. cocain are made to each eye, followed by one of adrenalin and one of 5 per cent. cocain to the injured eye. These should be spread over a period of about 5 minutes.

The lids are then wiped free from moisture, to enable a firm hold to be obtained by the surgeon's fingers.

\section{Technique of the Haab Magnet.}

1. Position of patient.-(a) If the patient can sit up.-The patient is seated on a chair of ordinary height with a back. The height of the patient is regulated with cushions until the eye is on a level slightly above that of the magnet point. The chair is drawn up as closely as possible to the magnet, the patient folds his arms and rests the forearms on the shelf attached to the magnet pillar (Fig. 1). This movement must be regulated by the surgeon, so as to avoid injury to the eye by bumping it against the magnet. 
(b) If injuries or general condition prevent the patient sitting up.This contingency is extremely unlikely in civil life, but has been met with frequently in war casualties.

The stretcher, with the patient on it, is placed on the operating table and the magnet is brought as close as possible to the head of the table, on the left side. The head-end of the stretcher is then moved laterally towards the magnet, so that one of the runners rests upon the arm shelf attached to the magnet pillar. The head of the

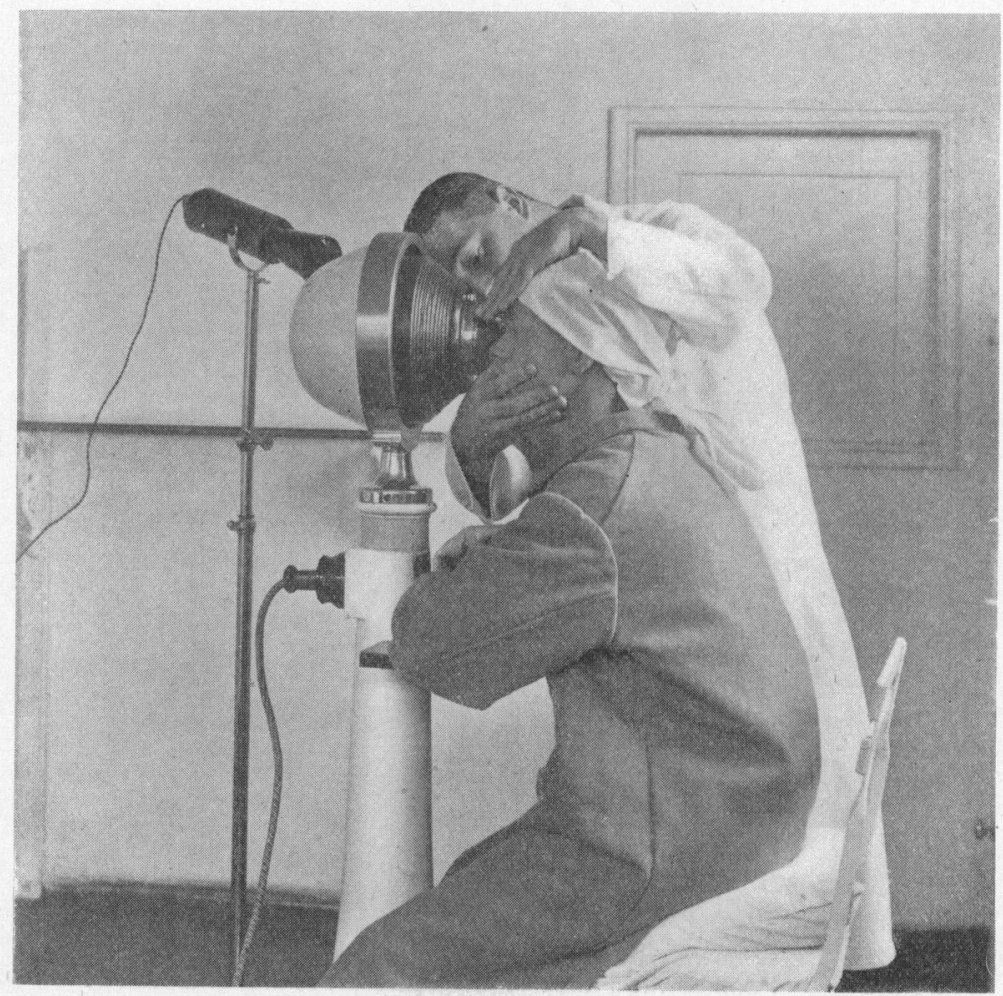

FIGURE 1.-Showing the general disposition of patient, operator, magnet, and light.

patient is elevated, by placing pillows under his head and shoulders, until he is in a semi-recumbent position, with the level of the injured eye a little above that of the magnet point. The head is then turned to face the magnet.

We always work with the magnet on the left side of the patient, irrespective of which eye is injured.

2. Position of Surgeon.- - The surgeon stands on the right side of the seated patient with his left foot and left shoulder somewhat behind the patient and his right foot and right shoulder slightly in advance. 
He rests the back of the patient's head in the hollow formed by the flexed elbow of the operator, supporting the head chiefly with the upper arm. This restrains any sudden movement of the head backwards, and enables the surgeon to bring the head of a nervous patient forward without undue effort.

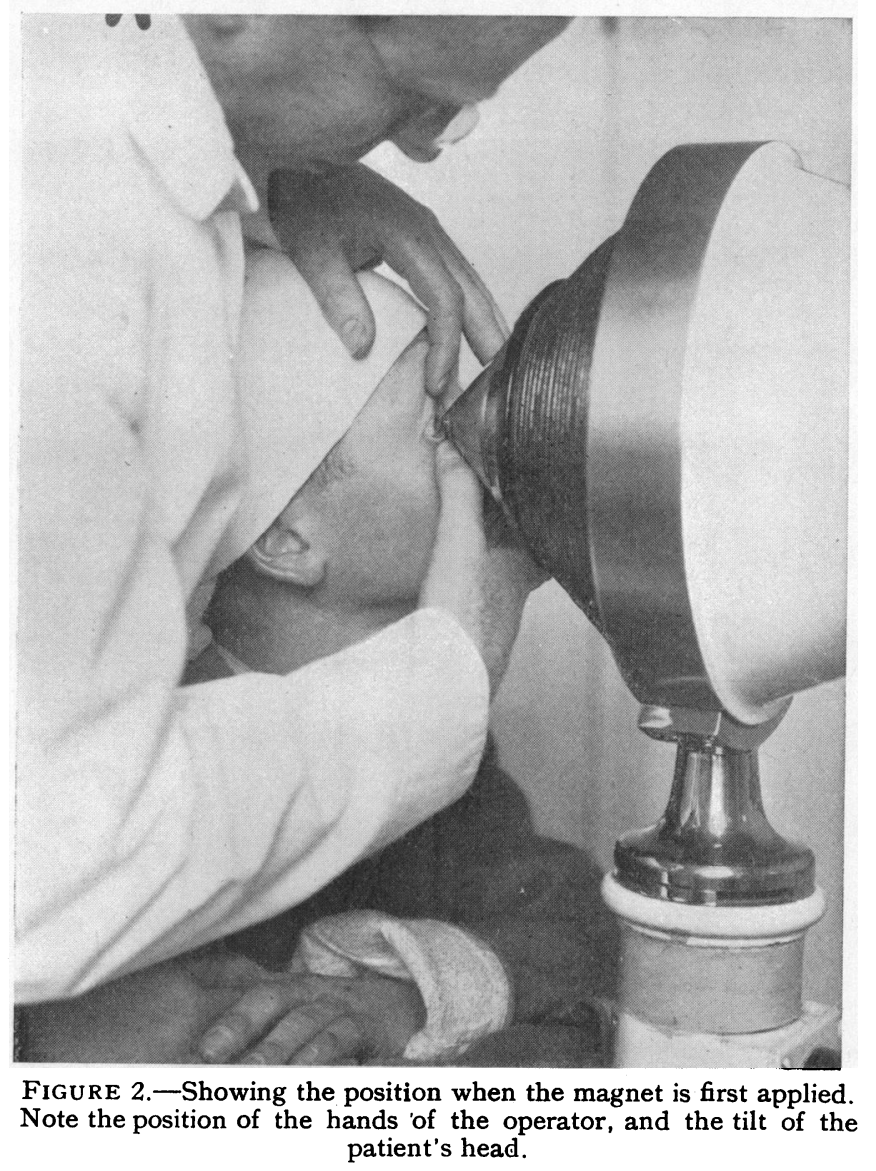

(a) Surgeon's hands. - The palm of the left hand is placed on the forehead of the patient above the injured eye; the forefinger retracts the upper eyelid and retains it in position by pressure against the upper margin of the orbit. This, also, by lifting the eyebrow controls the most powerful part of the orbicularis and corrugator supercilii muscles. The other fingers should remain outstretched and placed on the bevelled core of the magnet, so as to regulate the distance of the eye from the magnet and to prevent pressure of the magnet point upon the globe. The thumb of the left hand remains on the forehead of the patient. 
The fingers of the right hand grasp the lower jaw below, while the outstretched thumb retracts the lower eyelid, pressing against the lower margin of the orbit. The distal joint of the thumb is slightly flexed and the projection of this joint may be used to regulate further the distance of the eye from the magnet by contact with the bevelled core.

We know of no other grasp of the patient's head which gives such control and precision of movement. We have tried the method of standing behind the magnet and drawing the patient's head towards the point as illustrated in Haab's Atlas of Ophthalmic Surgery and have found it less satisfactory.

(b) Position of Surgeon's head.-The head should be inclined to his right shoulder, so that he views the patient's eye from the front as well as laterally and at the same time does not obstruct the light from the operating lamp. From this position any movement of the iris and often of the foreign body advancing through the vitreous can be observed.

3. Position of operating lamp.-The lamp is placed behind the magnet and on the patient's right side on the level of the magnet point. The beam of light is directed on the injured eye parallel to the bevel of the magnet core. The distance is regulated, so as to get the greatest concentration of light. The question of illumination is important, since a good light enables the surgeon to detect at once any movement of the iris.

(4) Manipulation of the patient's head.-The patient's head should not be advanced erect and facing directly forward to the magnet; if this be done, it will be found that the nose, to an extent dependent on its size, will come into contact with the bevelled core, and will prevent the head being advanced to the required position. Instead, the chin should be elevated, the head inclined slightly towards the same shoulder as the injured eye and rotated towards the opposite shoulder. Thus, in the case of a right eye, the head is tilted back, inclined to the right shoulder and rotated towards the left. The patient is instructed to keep both eyes widely open, to look at the magnet point, and to allow the surgeon to move his head freely in any direction. The head is then advanced until the magnet point comes into contact with the cornea just above its centre.

(5) Operation upon patient lying down.-In the cases which the general condition or injuries of the patient necessitate the recumbent position, or in which the operation with the Haab proves to be so painful that a general anæsthetic is required for its completion, the surgeon stands above the patient's head. The upper eyelid is retracted with the forefinger of the left hand and the lower eyelid with the forefinger or thumb of the right hand, the head and face being grasped between the two palms.

If the patient is under an anæsthetic and the whole weight of the 
head has to be supported, we have found it convenient to insert a Clarke's wire speculum between the eyelids. The pedal of the magnet has to be worked by the assistant at the direction of the operator.

The operating lamp in these circumstances is adjusted, so that the projector is over the patient's abdomen and the beam of light shines down on to the patient's face. It requires some care to secure the best illumination.

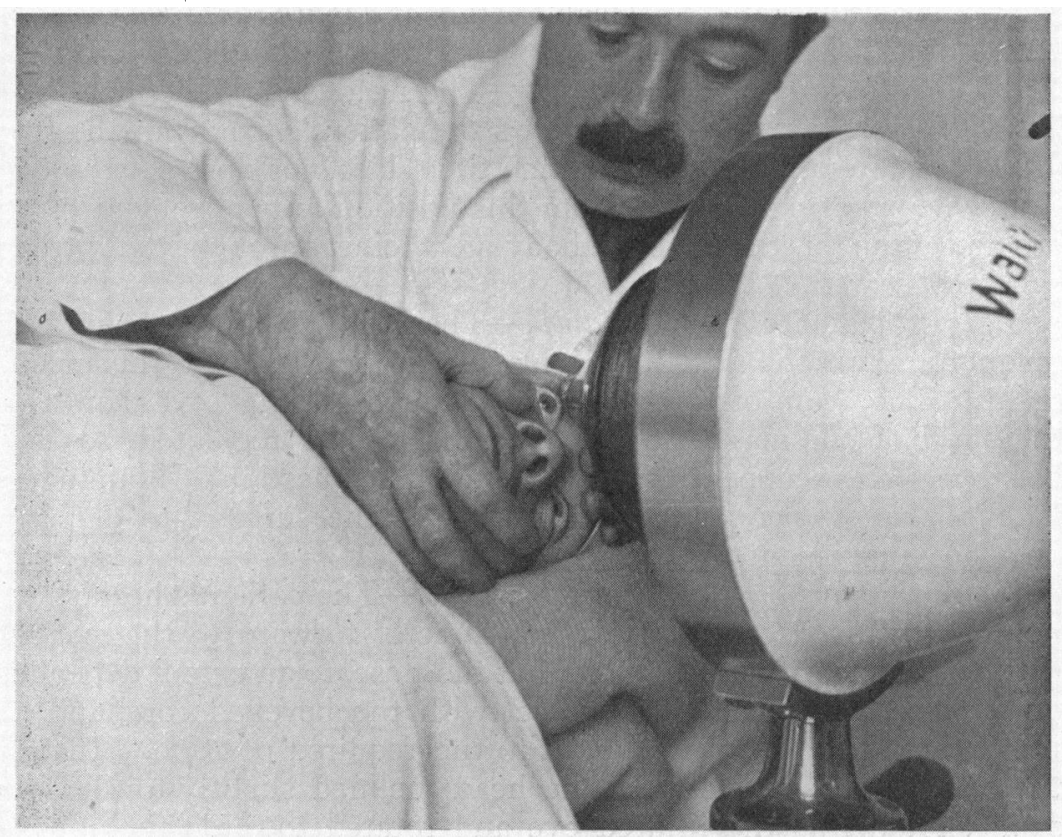

FIgURE 3.-Arrangement of the recumbent patient for operation upon the right eye.

The position for operating on the left eye is obtained by raising or rotating the patient's head.

The full strength of the current is switched on by depressing the pedal. It is important that at this moment the magnet point should actually be in contact with the cornea slightly above its centre. It is surprising how much power is lost if there is a space of only a few millimetres between the cornea and the point of the magnet.

The reason for this relative position of eye and magnet will be readily understood when we consider the forces acting on the foreign body, namely, the vertical pull of gravity and the horizontal pull of the magnet. The foreign body will follow the resultant of these two forces, and travel obliquely downwards instead of horizontally 
The Technique of the Haab and Small Magnets. 39

forwards. This is partly the reason why most foreign bodies present behind the lower part of the iris, also X-ray localisations show that in the majority of cases foreign bodies after penetrating the globe, sink to the lower part of the vitreous chamber.

The object of the sudden application of the full current is to disengage the foreign body from its bed (if it is resting on the retina),

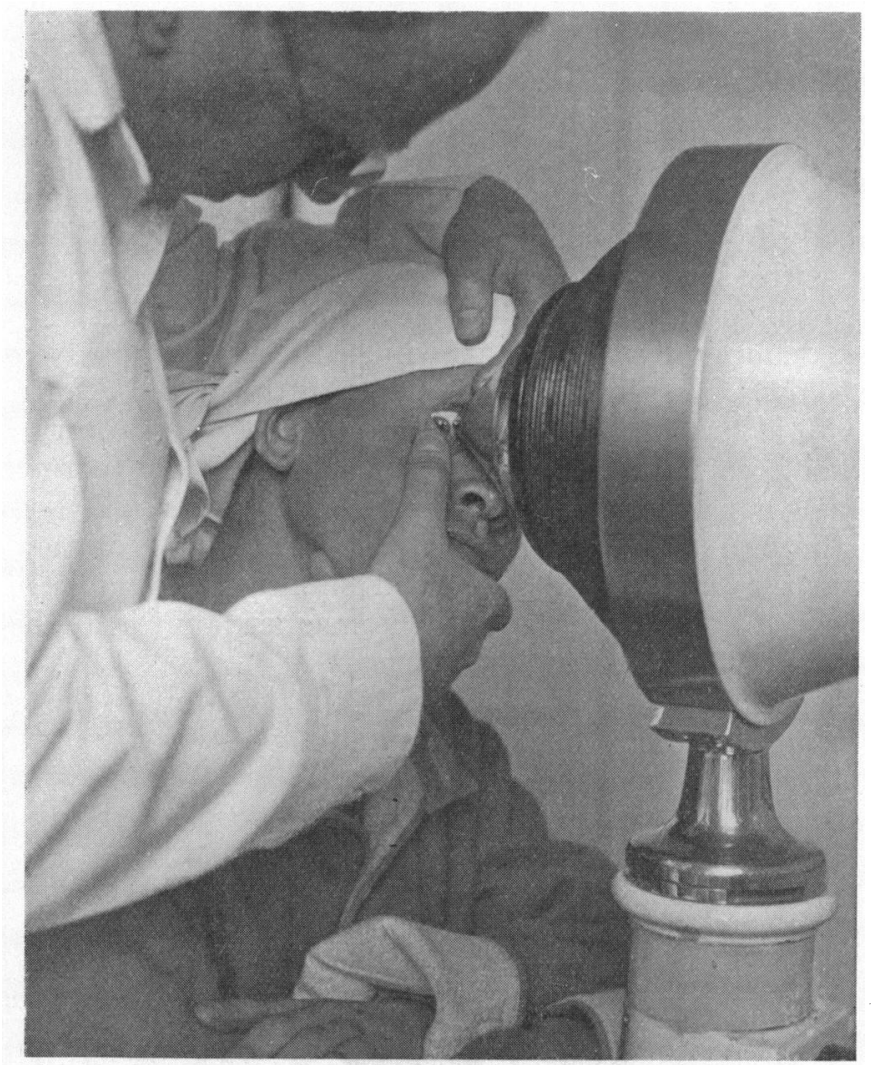

FIGURE 4.-Showing the position of applying the magnet for drawing the foreign body through the pupil after it has presented behind the iris below.

and to make it pass forward through the vitreous to the posterior surface of the lens as nearly as possible in the horizontal plane.

We have observed on several occasions foreign bodies advancing through the vitreous until they come into contact with the posterior surface of the lens, and then, slipping round the edge of the lens, suddenly appear quite free in the anterior chamber. This is the ideal result. 
If the force be gradually applied, either by the use of a rheostat, or by starting with the eye at some distance and gradually advancing the head until the cornea comes into contact with the magnet point, three dangers will be incurred, namely:- -the foreign body may be (1) dragged along the retina, (2) forced into the ciliary body, or (3) become entangled in the root of the iris. Usually, the foreign body shows its presence in the posterior chamber by pushing the iris before it.

When the foreign body presses on the iris, the patient experiences pain, and may state that he feels as if something were dragging on the eye. It is most important to notice the exact moment and position at which the bulging of the iris occurs; the moment, because the current must be cut off at once, to avoid entangling the foreign body in the substance of the iris, and the position, as this determines the subsequent manœuvres adopted to bring the foreign body through the pupil into the anterior chamber.

A very common position at which the foreign body presents itself is below. In such a case, after shutting off the current, the patient is told to look down, the head is tilted slightly forward, and the magnet point applied to the upper margin of the cornea. The current is then switched on again, and the foreign body slips forward through the pupil to the posterior surface of the cornea. The current is maintained, the head slightly withdrawn, and the patient told to look upwards until the magnet point is at the lowest part of the limbus.

The foreign body is thus drawn into the bottom of the angle of the anterior chamber, the most suitable position from which to remove it with the small magnet.

The above is a description of the simplest case. At any stage in the operation, difficulties may be encountered :

(1) Delay in the passage of the foreign body through the vitreous chamber.-Delay is most common in those cases in which the foreign body has been in the eye two or more days before the attempt at removal, and in which, consequently, it may be more or less fixed by exudate. Foreign bodies not infrequently appear in the anterior chamber coated with the exudate that has formed round them.

Almost always when a magnetic foreign body is present in the eye, the patient experiences some sensation of dragging from the first moment of application of the magnet. Even if no dragging sensation be felt, when the magnet is applied to the centre of the cornea, a very small magnetic foreign body may be present, and an attempt should be made to elicit the sensation by applying the point of the magnet to the sclera as far back as possible, above, below, and laterally. If the sensation of dragging be produced, efforts to bring forward the foreign body must be persisted in for a considerable time. It is in these cases that the method employed by Haab 
proves most useful, that of applying the force of the magnet in a series of jerks. The magnet is applied to the centre of the cornea, and the current rapidly switched on and off with the pedal, alternating this with a series of long pulls.

We have in some cases obtained ultimate success by persisting in this method for five or more minutes. It may be desirable to interrupt the attempt for some hours, if the magnet becomes overheated, in order to allow it to cool.

In obstinate cases, the foreign body may sometimes be loosened by applying the magnet to various points on the sclera, so as to pull the foreign body in various directions, and so to loosen it. In this way also a greater force may be exerted by placing the point closer to the foreign body. This manœuvre is similar to the loosening of a stake driven into the ground by lateral movements before removing it by a straight pull.

It may be noted that delay in the vitreous is much more likely to occur in those cases in which there is some element of infection.

The presence of infection in the eye frequently causes the formation of bands in the vitreous which are attached to the foreign body. It will then be found that the foreign body can be brought forward with the Haab magnet, but on breaking the current, it is drawn back again into the vitreous. In these cases, persistent attempts must be made to break the bands, or at all events to stretch them as much as possible, before proceeding to the second stage of removal with the small magnet. This should be done by varying the position of the eye and the patient's head, so as to cause the greatest possible amount of tension to be put on the bands when the magnet is applied.

(2) Entanglement of the foreign body in the iris.-When a foreign body is drawn forward, it frequently becomes more or less entangled in the iris. This shows itself by movement of the affected part of the iris towards the magnet-point. Such cases require the greatest amount of care in manipulation. It is imperative to disentangle the foreign body and to bring it forward into the anterior chamber; since if this is not done, any attempt to remove the foreign body with the small magnet is almost certain to end in disaster. It is improper to try to pull the foreign body through the iris; this will only impact it in the tissue.

The foreign body having shown its presence in the posterior chamber by bulging the iris forward, the current must be cut off immediately. We will suppose again that the foreign body has presented below in the right eye. The head of the patient is bent forward, the chin inclined to the left shoulder, and the magnet-point applied to the limbus up and out, so that the line of its pull shall be as far as possible in the plane of the iris, with as little forward drag as possible. The patient is at the same time told to look down 
and to the left. The current is then switched on, and after one or two attempts, the foreign body will probably be released and come forward. If this be not successful, it is generally possible to detach the foreign body from the iris by applying the magnet-point still further back on the sclera, so that the pull is actually behind the plane of the iris. Once the foreign body is detached, it can easily be brought forward into the anterior chamber.

Similar manœuvres may be adopted for foreign bodies presenting at other points, with an appropriate change of the relative positions of eye and magnet.

(3) The presence of a still corneal or corneo-scleral wound with prolapse of iris. - No attempt must be made to remove the foreign body through such a wound, or through any enlargement of that wound. The foreign body should be treated as indicated above and brought to rest as near the lower angle of the anterior chamber as possible. The subsequent treatment of such a condition will be described later.

(4) The presence of a very large corneal wound.-In these cases the presence of a large intra-ocular foreign body is indicated, and the chance of saving the eye is correspondingly slight. There is considerable danger that iris, ciliary body, lens, or vitreous may be dragged out in an attempt at removal of the foreign body. Any attempt with the Haab magnet must be begun very cautiously with the eye at some distance, and the effect closely observed.

At the first sign of the foreign body presenting behind the cornea all further attempts with the Haab must cease, and the foreign body should be removed with the small magnet-as a rule, through the corneal wound. The small magnet gives ample power and is much better under control.

(5) The presence of an unhealed scleral wound.-If the wound be large, the foreign body should be removed through this wound, with the same precautions as in the case of a large corneal wound, and the sclera be sutured. If the wound be small, the foreign body should be removed by way of the anterior chamber, as if no scleral wound existed. The wound in the sclera, made by a small foreign body, never gives sufficient room for the withdrawal of the foreign body with the magnet and consequently would have to be enlarged. Even were the hole sufficiently large, the chances of the foreign body engaging accurately in it are extremely slight. But in any case the conjunctiva, and, if necessary, the sclera, should be sutured carefully over the scleral wound after the foreign body has been removed.

\section{The Technique of the Small Magnet in the Removal of the Foreign Body from the Anterior Chamber.}

Having brought forward and deposited the foreign body in the lower angle of the anterior chamber, the next step is its removal 
from the eye. As a rule, this should be done under a general anæsthetic, for the eyes are often red and tender; the only exceptions are those cases in which the eye is white and, in the surgeon's opinion, the patient will be amenable to operation under local anæsthesia.

The patient is placed upon the operating table with the head supported by a cylindrical sand bag, which is adjusted in a comfortable position in the nape of the neck. A great deal of the success of the operation depends upon the manner in which the anæsthetic is given. It is most important that the patient should be "well under," and that the breathing should be free and not laboured. After the patient has been anæsthetised, the conjunctival sac is gently irrigated with normal saline solution at a temperature of $100^{\circ} \mathrm{F}$. For this purpose, the eyelids are everted, and special attention is paid to the lid-margins and to both canthi, as well as to the fornices.

The speculum is inserted, and a firm grasp of the conjunctiva and subconjunctival tissue is taken at, or slightly below, the horizontal meridian of the cornea just outside the limbus. The eye is rotated well downwards.

The corneal section is now made directly above, beginning at a point $3 \mathrm{~mm}$. below the limbus: that is, about half way between the limbus and the centre of the cornea. The point of the blade is directed straight towards the foreign body, and the section is completed by an onward thrust without lateral movement. A section made in this way will be of a valvular description, and on withdrawal of the keratome, the lips of the wound will come together and prevent the escape of aqueous.

The size of the incision is determined by the dimensions of the foreign body, and it may be necessary to carry the point of the keratome down almost to the angle of the anterior chamber. Allowance must be made for the fact that the opening on the deep surface of the cornea is smaller than that on the superficial surface.

The keratome is withdrawn fairly rapidly, but without any jerk, and precisely in the plane of its insertion. At the same moment the grasp of the conjunctiva is released. Provided that the patient is well under the anæsthetic, and that the section is made exactly as described, it will be found that little or no aqueous is lost. This end will be still more certainly attained if the weight of the speculum be taken off the eye by the assistant. Without loss of time, the small magnet is taken, and the point of the terminal is directed outside the cornea, and immediately over the foreign body. In this way the foreign body is attracted to the posterior surface of the cornea; by bringing the terminal upwards, still close to the cornea, the foreign body is drawn along the posterior surface and into the corneal section. The terminal is depressed on to the wound, and the foreign body thus withdrawn. During the whole 
manœuvre, if possible, the eye must not be touched with fixation forceps, or the aqueous will probably be lost. Any tendency of the iris to prolapse after the foreign body has been removed, is overcome by manipulation with the iris repositor.

In a certain number of cases, in spite of all care, the aqueous humour is completely lost. It is then impossible to withdraw the foreign body in the manner indicated above.

Two methods of dealing with the case are now open, the first of which is to fill the anterior chamber with normal saline solution, and then to remove the foreign body as before. This will be successful when the loss of aqueous is due to some cause which can be corrected, e.g., to slight squeezing, owing to the patient not being sufficiently anæsthetised, or to the weight of the speculum pressing on the globe.

The second method is carried out as follows:-

The eye is firmly seized with the fixation forceps in the horizontal meridian, and rotated well downwards. The magnet terminal, with the current off, is introduced into the corneal section, and passed straight down into the angle of the anterior chamber until it comes into actual contact with the foreign body. The current is then switched on, and the foreign body gently removed. Any tendency of the foreign body to hitch on the lips of the wound may be overcome by rotating the flattened magnet point so as to separate the lips of the incision. It will sometimes be found that on passing the terminal down along the posterior surface of the cornea, the lower pupillary border of the iris is pushed in front of it, and the magnet point cannot be brought into contact with the foreign body. Any attempt at removal then will only entangle the foreign body in the iris. The point must be insinuated in front of the iris by withdrawing it slightly, and sweeping it laterally and downwards with a movement following the curve of the pupillary border. If by chance the foreign body should become entangled in the iris, the current must be switched off and the terminal placed in a more favourable position before a fresh attempt is made, otherwise the iris will be torn away at its root. In our experience, cases in which an iridodialysis has been thus caused do badly.

In the numerous cases we have treated in this manner we have never had to do an iridectomy for prolapse of iris from the corneal section, either at the time of operation or later. In one or two cases only has there been adhesion of the iris to the corneal wound. These adhesions were easily detached with Lang's twin knives.

The foregoing description applies to uncomplicated cases ; various conditions may be present which make the operation more difficult :

(1) Complications due to the nature of the wound caused by the entrance of the foreign body.

(a) Presence of a prolapse of iris.- The procedure for removal of 
the foreign body is the same as that adopted in an uncomplicated case. After the foreign body has been removed, the prolapsed iris should be cut off in the usual way.

On no account should the iridectomy be performed first, and then an attempt made to remove the foreign body through the site of the prolapse or an enlargement thereof. If the wound extend past the limbus, it may be necessary to insert a conjunctival or scleral suture.

It may be noted that a wound of the sclera may have to be treated in the same way, and a prolapse of vitreous cut off. The presence of an unhealed wound makes the section more difficult, by reason of the lowered intra-ocular tension.

(b) The presence of a traumatic cataract.-If the foreign body can be seen in the lens, and the eye show no signs of infection, operation for removal of the foreign body should be deferred until such a time as the cataract is also ready for removal. If, however, the lens be considerably broken up, and coming forward into the anterior chamber, the foreign body should be brought forward with the Haab magnet and removed as described, and the operation completed by washing out as much soft lens matter as possible.

If the eye be infected, operation should be undertaken at once and the anterior chamber thoroughly irrigated with normal saline solution at a temperature of $100^{\circ} \mathrm{F}$. We have had several very gratifying results from such procedure.

(2) Complications due to infection of the eye.

(a) Presence of vitreous bands.-In the cases referred to under the technique of the Haab magnet, when vitreous bands prevent the foreign body coming forward freely, and the attempts with the Haab magnet have failed to free the foreign body entirely, difficulty will be experienced with the small magnet. Application of the point of the small magnet outside the cornea will be ineffectual; the terminal must be introduced into the anterior chamber and the end placed as near as possible to the spot where the foreign body presented. The current is then turned on and an attempt made to free the foreign body by gentle traction and lateral movements. It may be necessary to assist the magnet with some non-magnetic instrument, such as an iris repositor.

(b) Cases with exudate in the anterior chamber or hypopyon.The exudate, which often forms a cast of the anterior chamber, may come out with the foreign body, and in default of this, should be removed with iris forceps. After removal of the exudate, a rather illusory impression of the condition of the eye is produced by the clearing of the iris and pupil; but the subsequent course of such cases frequently proves disappointing.

If hypopyon be present, it should be removed by freely irrigating the anterior chamber with normal saline solution. 
The operation in every case is completed by placing a piece of sterile 1 per cent. atropine ointment in the conjunctival sac, and applying a pad and bandage.

The after-treatment of these cases is conducted on general lines, namely: frequent hot bathing and the use of atropine ointment, together with complete rest in bed.

We wish to express our sincere thanks to Colonel W. T. Lister, C.M.G., A.M.S., for his kind advice and encouragement, and for his assistance with the illustrations; and also to Lt.-Colonel Sidgwick, R.A.M.C., for allowing us to put on record the experience we have gained at his Hospital.

\title{
THE RING MAGNET.*
}

\author{
BY \\ T. HARrison Butler, M.D.Oxon. \\ ASSISTANT SURGEON TO THE BIRMINGHAM AND MIDLAND EYE HOSPITAL.
}

Until Professor Mellinger, of Basle, invented the ring magnet (Innenpolmagnet), the giant magnets in use by ophthalmic surgeons were all modifications of the Haab pattern. These consist of a central core of soft iron, surrounded by a bobbin of insulated copper wire. The core is prolonged into a cone which terminates in a point.

All these instruments have great polar magnetic power, but, as the lines-of-force radiate in all directions, the power is concentrated at the immediate pole, and rapidly falls off as the distance increasesin fact, diminishes as the square of the distance.

The divergence of the lines-of-force can be graphically shown by attaching a bunch of keys or iron filings to the pole of the magnet.

This feature diminishes the value of a magnet of the orthodox type for removing fragments of steel from the interior of the eye, and explains why a hand magnet is unable to extract these bodies unless it is brought into almost immediate contact with them.

A field of sufficient saturation can be obtained by the use of a giant magnet, such as a Haab, Volkmann, Schlosser, or Rollet.

Professor Mellinger has met the difficulty in a more scientific manner.

It is well known that when an electric current flows round a

* A paper read before the Midland Ophthalmological Societr on October 3rd. 1916. The new magnet in the operating theatre of the Birmingham and Midland Eye Hospital was shown to the Society, and the direction of the lines-of-force in the Haab and ring magnet was demonstrated by iron filings. 